\title{
Epigallocatechin-3-gallate protects against secondary osteoporosis in a mouse model via the Wnt/ $\beta$-catenin signaling pathway
}

\author{
JIANCHENG XI $^{1^{*}}$, QINGGUI LI ${ }^{2 *}$, XIAOBO LUO ${ }^{1}$, JINLONG LI $^{1}$, \\ LIXIN GUO $^{1}$, HAIBIN XUE ${ }^{1}$ and GUANGSEN WU ${ }^{1}$ \\ ${ }^{1}$ Department of Minimally Invasive Spinal Surgery, The 309th Hospital of The People's Liberation Army, Beijing 100091; \\ ${ }^{2}$ Department of Orthopedics, The Fourth Affiliated Hospital of Hebei University, Baoding, Hebei 071000, P.R. China
}

Received July 18, 2016; Accepted November 3, 2017

DOI: $10.3892 / \mathrm{mmr} .2018 .9437$

\begin{abstract}
Epigallocatechin-3-gallate (EGCG) is a polyphenolic compound extracted and isolated from green tea, which has a variety of important biological activities in vitro and in vivo, including anti-tumor, anti-oxidation, anti-inflammation and lowering blood pressure. The aim of the present study was to investigate the protective effect of EGCG against secondary osteoporosis in a mouse model via the $\mathrm{Wnt} / \beta$-catenin signaling pathway. Reverse transcription-quantitative polymerase chain reaction (RT-qPCR) and western blotting were used to analyze runt-related transcription factor 2 and osterix mRNA expression, and the protein expression of cyclin D1, Wnt and $\beta$-catenin, and suppressed peroxisome proliferator-activated receptor $\gamma$ protein expression. The protective effect of EGCG against secondary osteoporosis was examined and its potential mechanism was analyzed. Treatment with EGCG significantly decreased serum calcium, urinary calcium, body weight and body fat, and increased leptin levels in mice with secondary osteoporosis. In addition, EGCG treatment significantly inhibited the structure score of articular cartilage and cancellous bone in proximal tibia metaphysis in mice with secondary osteoporosis. Treatment also significantly decreased alkaline phosphatase activity, runt-related transcription factor 2 and osterix mRNA expression. EGCG also significantly induced the protein expression of cyclin D1, Wnt and $\beta$-catenin, and suppressed peroxisome proliferator-activated receptor $\gamma$ protein expression in mice with secondary osteoporosis. Taken together, these results suggest that EGCG may be a possible new drug in clinical settings.
\end{abstract}

Correspondence to: Dr Qinggui Li, Department of Orthopedics, The Fourth Affiliated Hospital of Hebei University, 212 Yuhua East Road, Baoding, Hebei 071000, P.R. China

E-mail: xijcjcbj@163.com

*Contributed equally

Key words: epigallocatechin-3-gallate, secondary osteoporosis, Wnt, $\beta$-catenin

\section{Introduction}

Osteoporosis is a type of systemic bone disease that is characterized by low bone mass, microstructural damage of the bone, increased bone fragility and potential bone fracture (1). Osteoporosis can be divided into two categories, primary type and secondary type (2). The former is further divided into postmenopausal (type I), senile (type II) and idiopathic osteoporosis disease. The latter refers to osteoporosis induced by diseases that influence bone physiology or by drugs, such as osteoporosis induced by long-term high-dose glucocorticoid treatment (3). In general, postmenopausal (type I) osteoporosis occurs within 5-10 years following the menopause in women, senile osteoporosis develops in individuals aged $>70$ years old and idiopathic osteoporosis is predominantly observed in adolescents; however, its pathogenesis is unknown (3). Due to the wide application of glucocorticoids in clinical practice, the incidence of glucocorticoid-induced osteoporosis has continuously risen in recent years, and as such, it is now considered the third most common type of osteoporosis, following postmenopausal and senile osteoporosis (4).

The role of Wnt signaling in bone metabolism has become a key area of interest in recent years. Previous studies have demonstrated that Wnt can directly affect the pluripotent precursor cell differentiation process into bone cells $(4,5)$. The stable expression of Wnt 1 and Wnt3a can promote the proliferation of osteocytes and induce alkaline phosphatase (ALP) activity, which is the early stage marker of osteoblast differentiation (6). However, apart from ALP, the other relevant markers for osteoblast differentiation, including runt-related transcription factor 2 (Runx2), osteocalcin (OC) and type I collagen, are not markedly affected, which indicates that Wnts can promote the growth of precursor osteoblasts and promote osteoblast differentiation at the early stage (7).

$\beta$-catenin serves an important role in the Wnt signaling pathway. The bone stem cell lineage can differentiate into osteoblasts, adipocytes and chondrocytes; it can also be differentiated into osteoblasts via the action of bone morphogenetic protein 2 (BMP-2). During this process, BMP-2 can upregulate $\beta$-catenin (8). The overexpression $\beta$-catenin in the $\mathrm{C} 3 \mathrm{H} 10 \mathrm{~T} 1 / 2$ cell line can increase the expression and activity of ALP; however, it had no significant effect on OC, which is a differentiation marker of osteoblasts during the late stage (9). 
This suggests that $\beta$-catenin may serve a role in precursor osteoblast and osteoblast proliferation and differentiation, and that it may be regulated by BMP-2 (10).

Tea contains a large amount of polyphenolic compounds, which are collectively known as tea polyphenols, accounting for $18-36 \%$ of the dry weight of tea; a number of studies have shown that polyphenols have a strong antioxidant capacity and serve as natural food antioxidant additives $(11,12)$. A previous study investigating the chemical compositions of tea polyphenols demonstrated that the main components of tea polyphenols are catechins, accounting for 70 to $80 \%$ of total polyphenols, which mainly included catechin (C), epicatechin $\mathrm{Su}$ (EC), epicatechin gallate (ECG), epigallocatechin-3-gallate (EGCG) and other active substances; EGCG occupied the highest content percentage of $\sim 50$ to $60 \%$ of total catechins (12). A large number of studies have revealed that EGCG has biological activities including anti-cancer, anti-mutation, the prevention and treatment of cardiovascular diseases, and regulating the endocrine and immune systems; it also has inhibitory activity associated with metabolic enzymes, which have a marked impact on the liver detoxification function of the body $(13,14)$. The aim of the present study was to investigate the potential anti-osteoporosis effects of EGCG in secondary osteoporosis and the potential underlying mechanism in a mouse model.

\section{Materials and methods}

Animal experiments. The animal protocol was approved by the Committee on the Ethics of Animal Experiments of The 309th Hospital of The People's Liberation Army (Beijing, China). A total of 22 6-week-old male C57BLKS/J mice (weight, 20-22 g) were purchased from Beijing Vital River Laboratory Animal Technology Co., Ltd. (Beijing, China) and were given free access to food and water. They were caged individually under a controlled temperature $\left(23-25^{\circ} \mathrm{C}\right)$ and humidity $(50-60 \%)$ with a $12 \mathrm{~h}$ artificial light/dark cycle. The present study used a dexamethasone-induced model of osteoporosis, as described previously (4). Mice were divided into 3 groups: The control $(n=6)$, model $(n=8)$ and EGCG $(0.5 \mathrm{mg} / \mathrm{kg} /$ day; IP, $n=8 ;$ Fig. 1$)$ groups. In the model and EGCG groups, mice were injected with dexamethasone $(5 \mathrm{mg} / \mathrm{kg} /$ day $)$ into the intraarticular space of the right knee for 4 weeks to establish the osteoporosis model (15). In EGCG groups, mice were injected with $0.5 \mathrm{mg} / \mathrm{kg} /$ day of EGCG for 4 weeks. At 0 and 4 week, body weight was record, and blood was were collected from tail vein and used to measure body fat using a commercial kit (A042-2; Nanjing Jiancheng Bioengineering Institute, Nanjing, China).

Body weight and body fat measurements. The mice were anesthetized with an intraperitoneal injection of $30 \mathrm{mg} / \mathrm{kg}$ pentobarbital sodium and body weight was measured at the 0 and 4 week time points of EGCG treatment. In addition, Duel-Energy X-ray Absorptiometry (Lunar Prodigy; GE Healthcare, Chicago, IL, USA) was used to measure body fat at the 0 and 4 week time points of EGCG treatment.

Serum calcium, urinary calcium, and bone and energy metabolism. The mice were anesthetized with an intraperitoneal injection of $30 \mathrm{mg} / \mathrm{kg}$ pentobarbital sodium. Then, blood was collected from the tail vein and blood was transferred to tubes. A TechniconSMAC (Technicon Instruments Corp, Tarrytown, NY, USA) determined the serum calcium level. Urinary calcium/creatinine (cat no. C004-2) and ALP activity (cat no. A059-2) was measured using ELISA kits obtained from Nanjing Jiancheng Bioengineering Institute (Nanjing, China). The leptin serum concentrations were assayed at Novartis International AG (Basel, Switzerland) using a Luminex $200^{\mathrm{TM}}$ Multiplexing Instrument.

Histomorphological analysis. Following treatment with EGCG, mice were anesthetized with an intraperitoneal injection of $30 \mathrm{mg} / \mathrm{kg}$ pentobarbital sodium and sacrificed using decollation. Femoral head tissue was dissected by the axial plane and were fixed in $4 \%$ formaldehyde at room temperature for $>24 \mathrm{~h}$. These bone samples were then decalcified in $10 \%$ ethylene diamine tetraacetic acid solution for 15 days at room temperature and embedded in paraffin. Samples were cut into $4 \mathrm{~mm}$ thick sections and stained with hematoxylin and eosin at $5 \mathrm{~min}$ at room temperature. The articular cartilage (AC) and cancellous bone in proximal tibia metaphysis (PTM) were then observed using a fluorescent microscope (x20; Zeiss Axioplan 2-300, Carl Zeiss MicroImaging) using the Mankin histological grading system, as described previously (16).

Reverse transcription-quantitative polymerase chain reaction (RT-qPCR). Total RNA was prepared using TRIzol Reagent (Gibco; Thermo Fisher Scientific, Inc., Waltham, MA, USA) from femoral head tissue. Total RNA was then reverse transcribed using a Reverse Transcription kit (Applied Biosystems; Thermo Fisher Scientific, Inc.). RT-qPCR was conducted using a Rotor-Gene 3000 System (Corbett Life Science; Qiagen, Inc., Valencia, CA, USA) and the SYBR Green PCR Master Mix Reagent kit (Qiagen, Inc.). The primers used were as follows: Runx2 forward, 5'-TGTCATGGCGGGTAACGATG-3' and reverse, 5'-CCCTAAATCACTGAGGCGGT-3'; OSX forward, 5'-CCTCTGCGGGACTCAACAAC-3' and reverse, 5'-AGC CCATTAGTGCTTGTAAAGG-3'; and GAPDH forward, 5'-CTATAAATTGAGCCCGCAGC-3' and reverse, 5'-GAC CAAATCCGTTGACTCCG-3'. The thermocycling conditions were: $95^{\circ} \mathrm{C}$ for $10 \mathrm{~min}$, then 40 cycles of $95^{\circ} \mathrm{C}$ for $30 \mathrm{sec}, 60^{\circ} \mathrm{C}$ for $45 \mathrm{sec}$, followed by $72^{\circ} \mathrm{C}$ for $30 \mathrm{sec}$. The expression was quantified using $2^{-\Delta \Delta \mathrm{Cq}}$ method (17).

Western blotting. Total proteins were extracted from femoral head tissue using Radioimmunoprecipitation Assay Lysis Buffer (Beyotime Institute of Biotechnology, Jiangsu, China) and measured using an Enhanced Bicinchoninic Acid Protein Assay kit (Beyotime Institute of Biotechnology). $50 \mu \mathrm{g}$ proteins were separated by $10 \%$ SDS-PAGE and then transferred to a polyvinylidene difluoride membrane (EMD Millipore, Billerica, MA, USA). Following incubation in 5\% skim milk powder and TBST in $0.1 \%$ Tween-20 for $1 \mathrm{~h}$ at room temperature, the membrane was hybridized with anti-Cyclin D1 (cat no. sc-717; 1:1,000; Santa Cruz Biotechnology, Inc., Dallas, TX, USA), anti-Wnt (sc-136163; 1:1,000; Santa Cruz Biotechnology, Inc.), anti- $\beta$-catenin (cat no. sc-7199; 1:1,000; Santa Cruz Biotechnology, Inc.), anti-peroxisome proliferator-activated receptor (PPAR)- $\gamma$ (cat no. sc-81152; 1:1,000; Santa Cruz Biotechnology, Inc.) and anti-GAPDH 


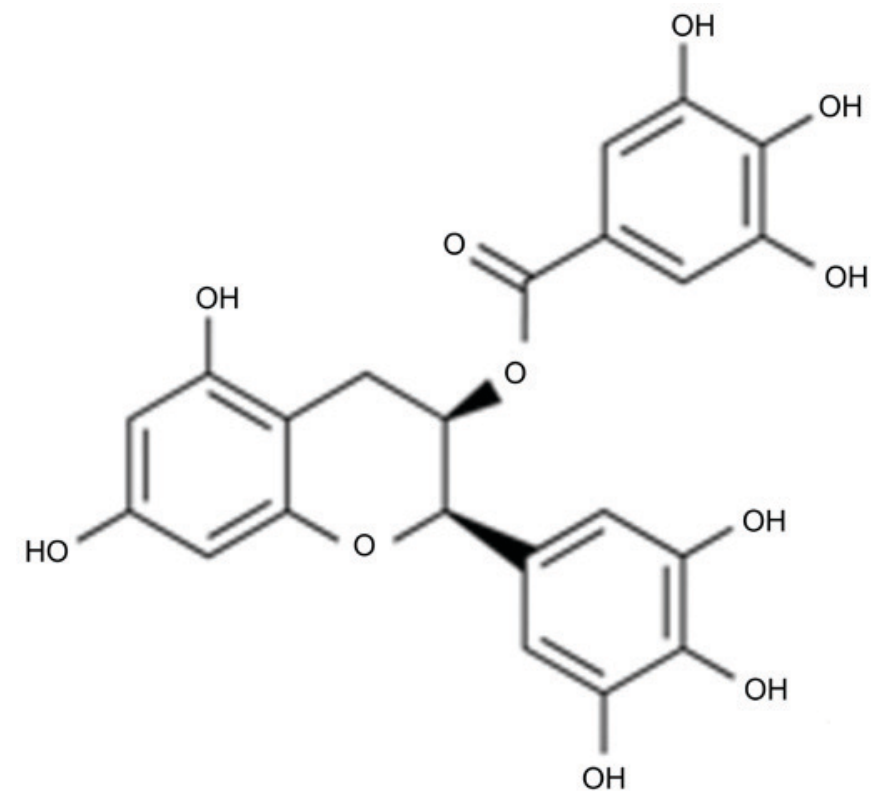

Figure 1. Chemical structure of epigallocatechin-3-gallate.

(cat no. sc-25778; 1:5,000;) primary antibodies overnight at $4^{\circ} \mathrm{C}$. The membrane was then incubated with anti-rabbit or anti-mouse horseradish peroxidase-conjugated secondary antibodies (cat no. sc-2004 or sc-2005, respectively; Santa Cruz Biotechnology, Inc.) at $37^{\circ} \mathrm{C}$ for $1 \mathrm{~h}$. Proteins were detected with an enhanced chemiluminescence reagent (Amersham; GE Healthcare) and analyzed using Image-Pro Plus version 6.0 software (Media Cybernetics, Inc., Rockville, MD, USA).

Statistical analysis. Data are presented as the mean \pm standard error of the mean of three independent experiments. The results were analyzed using SPSS 17.0 software (SPSS, Inc., Chicago, IL, USA), and a one- or two-way analysis of variance with a Tukey post hoc test were performed to evaluate the data. $\mathrm{P}<0.05$ was considered to indicate a statistically significant difference.

\section{Results}

EGCG protects against lipid metabolism. The effect of EGCG on lipid metabolism was evaluated in a mouse model of secondary osteoporosis. Following the 4 week treatment period, the body weight and body fat content in the secondary osteoporosis mouse model group were markedly higher than those of control group (Fig. 2). Treatment with EGCG for 4 weeks effectively reduced this secondary osteoporosis-induced increase in body weight and body fat content (Fig. 2).

EGCG protects against secondary osteoporosis. Under experimental conditions, the levels of leptin, calcium, calcium activity and urine calcium/creatinine $(\mathrm{Ca} / \mathrm{Cr})$ were detected. As shown in Fig. 3A, the leptin level was significantly decreased in secondary osteoporosis mouse model group at 4 weeks when compared with the control group. In addition, the levels of calcium, calcium activity and urine $\mathrm{Ca} / \mathrm{Cr}$ in the secondary osteoporosis mouse model group were significantly increased when compared with the control group (Fig. 3B-D).
Treatment with EGCG effectively enhanced the level of leptin and inhibited the levels of calcium, calcium activity and urine $\mathrm{Ca} / \mathrm{Cr}$ when compared with the model group (Fig. 3).

EGCG protects bone structure and bone turnover. To determine the anti-osteoporosis effects of EGCG associated with bone structure and bone turnover, the AC and PTM structure scores were analyzed. As shown in Fig. 4, the AC and PTM structure scores in the secondary osteoporosis mouse model group were significantly greater than those of the control group. EGCG treatment significantly decreased the AC and PTM structure scores when compared with the model group (Fig. 4).

EGCG decreases secondary osteoporosis-induced ALP activity, and Runx2 and Sp7 transcription factor osterix (OSX) mRNA expression. To determine whether ALP, Runx2 and OSX are involved in the effects of EGCG on osteoporosis, ALP, Runx 2 and OSX mRNA expression were measured using RT-qPCR. A significant increase in ALP, Runx 2 and OSX mRNA expression was observed in the secondary osteoporosis mouse model group when compared with the control group (Fig. 5). In addition, treatment with EGCG significantly reduced this secondary osteoporosis-induced increase in ALP, Runx 2 and OSX mRNA expression (Fig. 5).

EGCG decreases secondary osteoporosis-induced Runx2, $O S X$ and PPAR protein expression. The effects of EGCG on Runx2, OSX and PPAR $\gamma$ protein expression were further determined by western blotting (Fig. 6A). Osteoporosis significantly induced Runx2 (Fig. 6B), OSX (Fig. 6C) and PPAR $\gamma$ (Fig. 6D) protein expression in the secondary osteoporosis mouse model group when compared with the control group. In addition, treatment with EGCG significantly suppressed this osteoporosis-induced increase in Runx2, OSX and PPAR $\gamma$ protein expression.

EGCG increases Wnt, $\beta$-catenin and cyclin D1 protein expression in secondary osteoporosis. To investigate the role of Wnt, $\beta$-catenin and cyclin D1 in the effect of EGCG on osteoporosis, western blotting was performed (Fig. 7A). Wnt (Fig. 7B), $\beta$-catenin (Fig. 7C) and cyclin D1 (Fig. 7D) protein expression in the secondary osteoporosis mouse model group was significantly lower than that of the control group. However, treatment with EGCG significantly increased Wnt, $\beta$-catenin and cyclin D1 protein expression when compared with the secondary osteoporosis mouse model group.

\section{Discussion}

Osteoporosis is a common degenerative disease that primarily causes increased bone fragility and reduced bone density, which eventually leads to bone fracture (18). China has the greatest aging population in the world, of which at least 90 million people suffer from osteoporosis; it is expected that the number of patients with osteoporosis will increase to 221 million by the year 2050 (19). As osteoporosis severely affects the health of the elderly, identifying prevention strategies and treatments has become an major public health concern. The results of the present study demonstrated that 
A

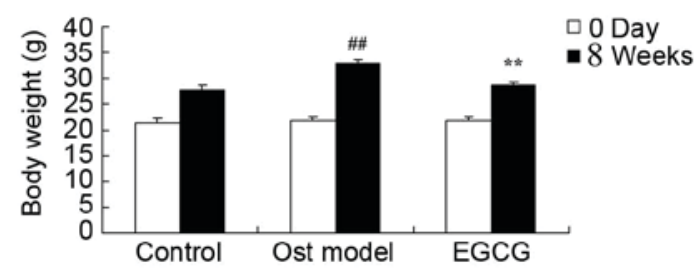

B

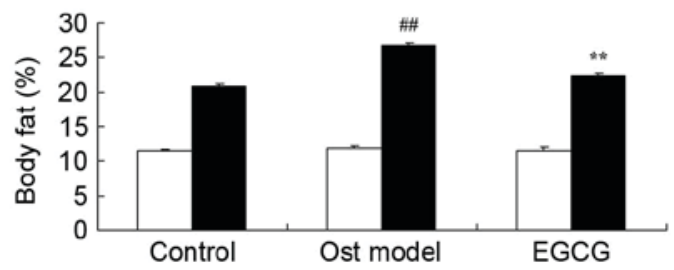

Figure 2. EGCG protects against adverse lipid metabolism. Following 4 weeks of EGCG treatment the (A) body weight and (B) percentage of body fat in mice with secondary osteoporosis was significantly decreased. ${ }^{\# \#} \mathrm{P}<0.01$ vs. control group; ${ }^{* *} \mathrm{P}<0.01$ vs. osteoporosis model group. Control, control group; Ost model, secondary osteoporosis model group; EGCG, epigallocatechin-3-gallate group.
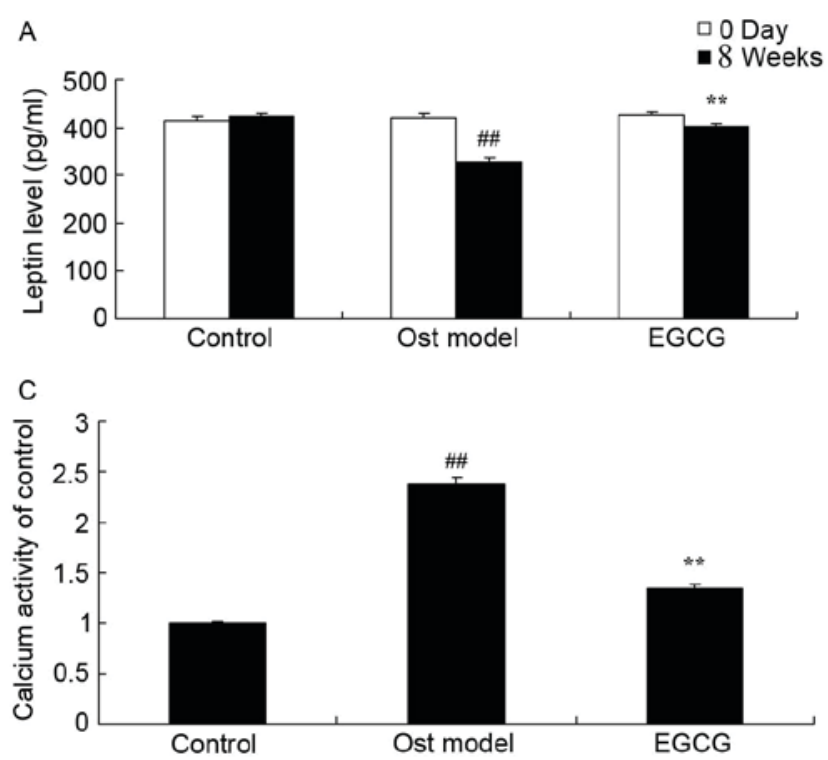

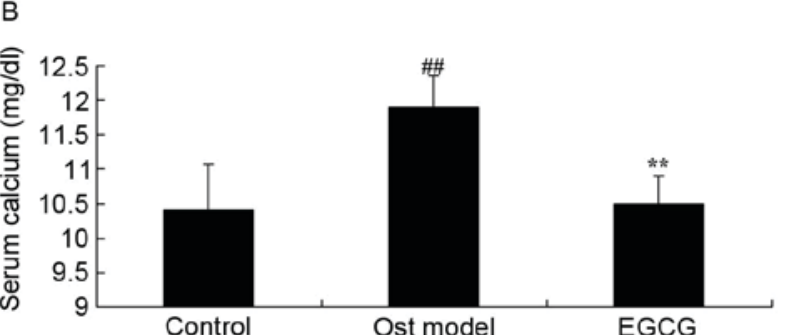

D

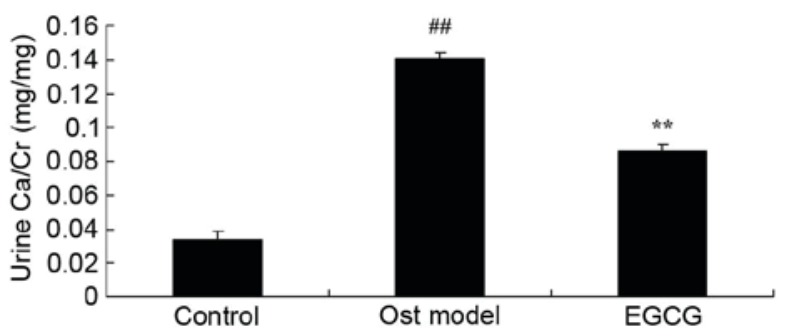

Figure 3. EGCG protects against secondary osteoporosis. EGCG treatment was protective of (A) the levels of leptin, (B) the calcium level, (C) calcium activity and (D) urine $\mathrm{Ca} / \mathrm{Cr}$ in a mouse with secondary osteoporosis. ${ }^{\# \#} \mathrm{P}<0.01$ vs. control group; ${ }^{* *} \mathrm{P}<0.01$ vs. osteoporosis model group. Control, control group; Ost model, secondary osteoporosis model group; EGCG, epigallocatechin-3-gallate group; $\mathrm{Ca} / \mathrm{Cr}$, calcium/creatinine.

EGCG is protective against adverse lipid metabolism, and bone structure and turnover in mice with secondary osteoporosis, which suggests that EGCG may exert anti-osteoporosis effects.

Osteoblasts are mainly derived from bone marrow mesenchymal stem cells, the differentiation process of which is dependent on the regulation of a number of transcription factors and cytokines (20). Bone marrow mesenchymal stem cells are pluripotent stem cells that can differentiate into osteoblasts, chondrocytes and adipocytes (21). A previous study revealed that when the cells have high expressions of Runx 2 and OSX, mesenchymal stem cells are differentiated into osteoblasts (21). However, when these cells highly express sex-determining region Y-box 9, mesenchymal stem cells are differentiated into chondrocytes, and when they have a high expression of PPAR, they differentiate into chondrocytes (22). The results of the present study demonstrated that EGCG treatment significantly reduced ALP, Runx2 and OSX mRNA expression, and suppressed Runx 2 and OSX protein expression in a mouse model of secondary osteoporosis.

Marrow fat cells are derived from mesenchymal stem cells. PPAR $\gamma$ mediates marrow mesenchymal stem cell differentiation into fat cells. PPAR $\gamma$ is a subtype of PPARs, which is a ligand-activated nuclear transcription factor that is involved in cell differentiation, growth and apoptosis (23). In addition, PPAR $\gamma$ mediates the adipogenesis of marrow mesenchymal stem cells (24). A higher expression of PPAR $\gamma$ protein in mice indicates a significant increase in bone turnover and lower osteogenic differentiation in marrow (25). These data demonstrated that EGCG significantly inhibited PPAR $\gamma$ protein expression in secondary osteoporosis. Lee et al (26) suggested that EGCG may suppress lipid deposition through PPAR $\gamma$ and the $\mathrm{WNT} / \beta$-catenin signaling pathway.

Runx 2 gene expression is regulated by a variety of hormones, cytokines and endogenous active substances (25). A number of signaling pathways have been shown to participate in the regulation of Runx2 expression or activity (25). The Wnt-low-density lipoprotein receptor-related protein 5 (LRP5)- $\beta$-catenin signaling pathway serves an important role in osteoblast proliferation and differentiation (27). Wnt and LRP5/6 are combined to form a complex receptor that induces $\beta$-catenin activation and migration to the nucleus; $\beta$-catenin in the nucleus binds the lymphoid enhancer-binding factor-1/T cell factor 1 transcription factors to form a compound that activates the transcription of the Run $x 2$ gene (28). The addition of Wnt inhibitors can inhibit the proliferation and differentiation 
A

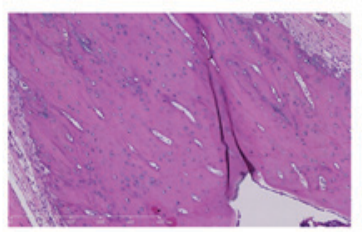

Control

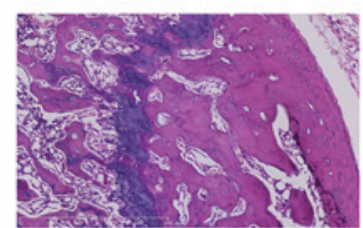

Ost model

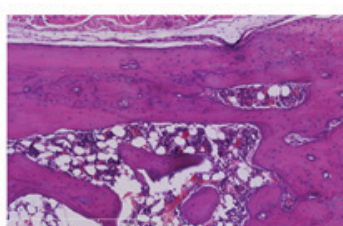

EGCG
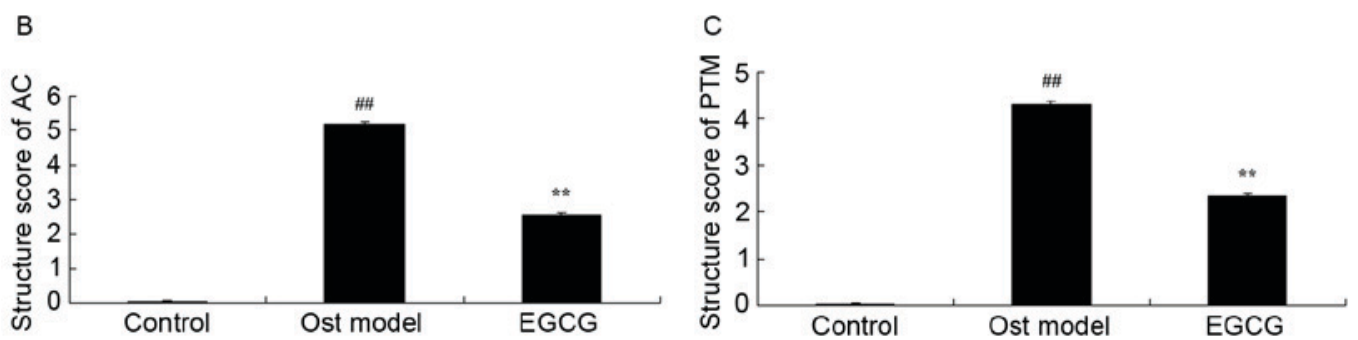

Figure 4. EGCG protects bone structure and turnover in secondary osteoporosis. (A) Hematoxylin and eosin staining of bone tissues to analyze the (B) AC and (C) PTM scores in mice with secondary osteoporosis. EGCG protected mice with secondary osteoporosis against adverse (B) bone structure of AC and (C) PTM of bone turnover in a mouse model of secondary osteoporosis. Magnification, $\mathrm{x} 100 .{ }^{\# \#} \mathrm{P}<0.01$ vs. control group; ${ }^{* * *} \mathrm{P}<0.01$ vs. osteoporosis model group. Control, control group; Ost model, secondary osteoporosis model group; EGCG, epigallocatechin-3-gallate group; AC, articular cartilage; PTM, proximal tibia metaphysis.

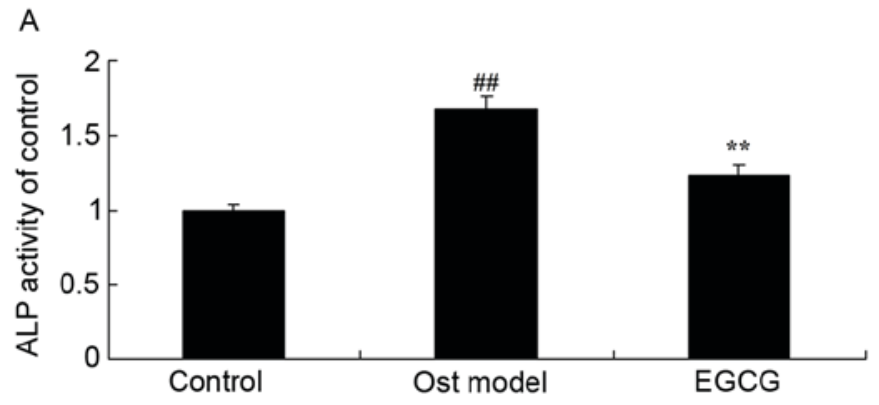

B

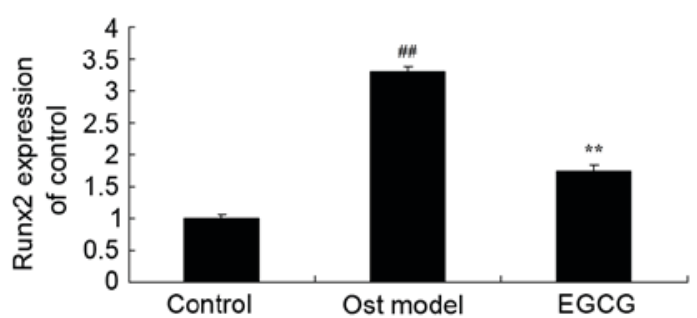

C

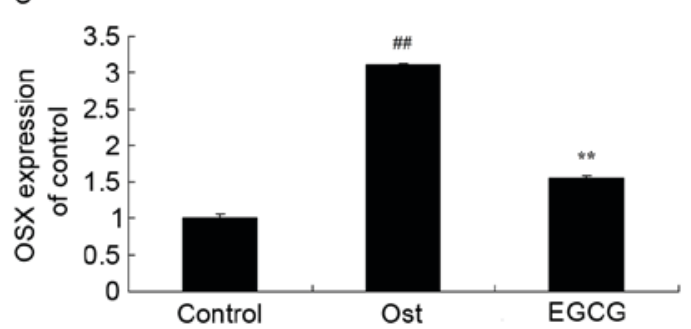

Figure 5.EGCG restores ALP activity, and Runx2 and OSX mRNA expression. Secondary osteoporosis significantly increased (A) ALP activity, and (B) Runx2 and (C) OSX expression, however, EGCG treatment decreased these levels in a mouse model of secondary osteoporosis. ${ }^{\# \#} \mathrm{P}<0.01$ vs. control group; ${ }^{* *} \mathrm{P}<0.01 \mathrm{vs}$. osteoporosis model group. Control, control group; Ost model, secondary osteoporosis model group; EGCG, epigallocatechin-3-gallate group; ALP, alkaline phosphatase; Runx2, runt-related transcription factor 2; OSX, Sp7 transcription factor osterix.

of osteoblasts. In mice without the secreted frizzled-related protein 1 gene, the Wnt signaling pathway can be significantly activated, Runx 2 promoter activity is increased and the mRNA content of the Runx 2 gene is increased; this also indicates that the Wnt- $\beta$-catenin signaling pathway may regulate the expression of Runx2 (29). Previous studies have revealed that BMP2 can activate mothers against decapentaplegic homolog 1/5 (Smad1/5) in osteoblasts, and induce Smad4 activation and nuclear translocation, thereby activating Runx $2(9,30)$. Taken together, the experimental results of the present study demonstrated that EGCG significantly induced $\beta$-catenin and Wnt3a protein expression in mice with secondary osteoporosis. Thus, $\beta$-catenin/Wnt may regulate the expression as well as the activities of PPAR $\gamma$ in secondary osteoporosis treated with EGCG.

CyclinD1 synthesis and expression is dependent on growth factors, and following the removal of growth factors, CyclinD1 synthesis is terminated immediately (31). Therefore, it is regarded to have the effect of growth factor sensors; namely, the growth factor-induced signal is associated with the regulation of the cell cycle. Cyclin D1, cyclin dependent kinase 4 (CDK4) and CDK6 combine to form a CDK4/6-CyelinDl complex that promotes the G1 phase of the cell cycle; CyclinD1 is a key protein for the cell proliferation signals of G1 phase (32). The present study revealed that EGCG treatment significantly induced Cyclin 

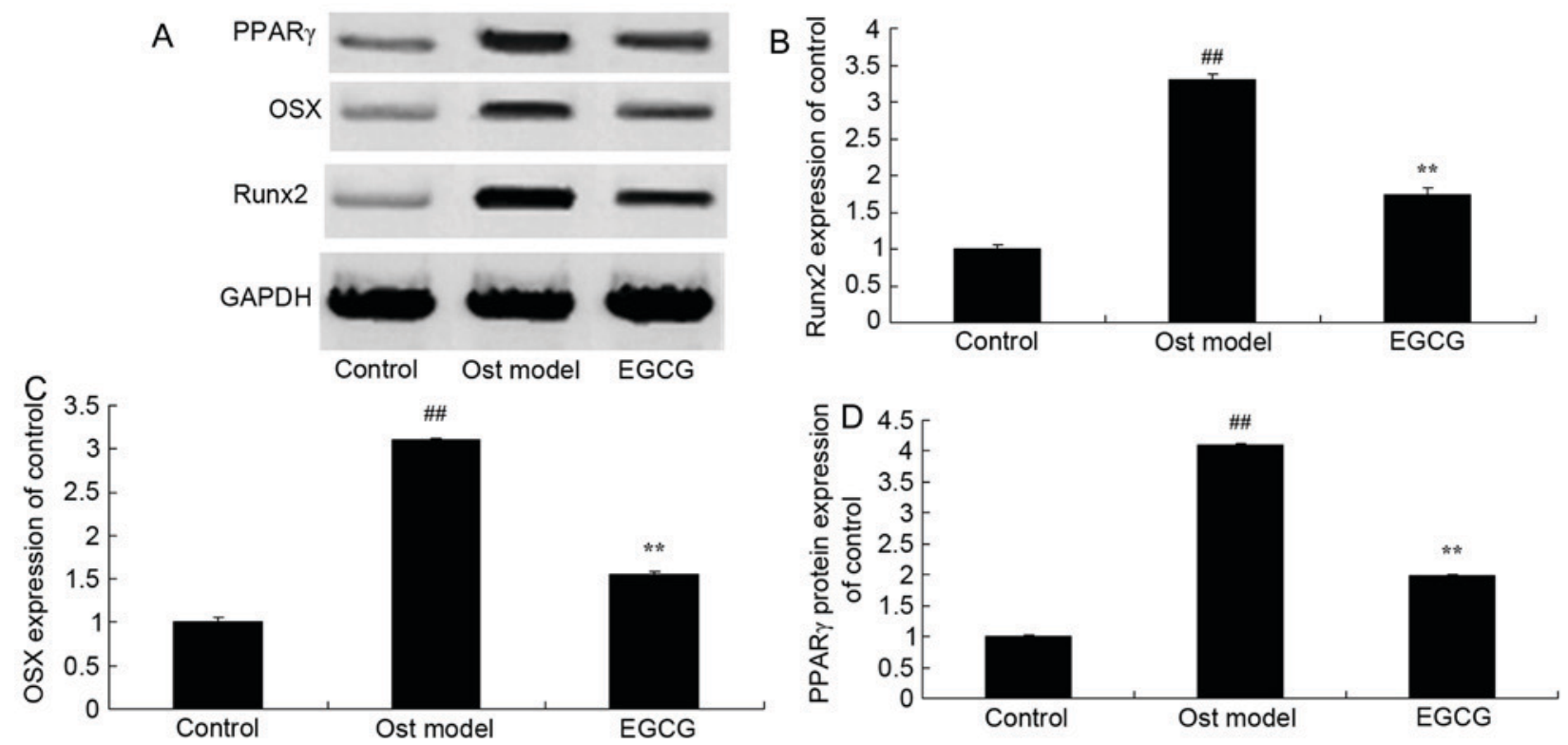

Figure 6. EGCG and Runx2, OSX and PPAR $\gamma$ protein expression. EGCG protects against Runx2 protein expression as shown by (A) western blot analysis. (B) Runx2, (C) OSX and (D) PPAR $\gamma$ protein expression increased in the mouse model of secondary osteoporosis, however, treatment with EGCG reversed this effect and decreased the expression of these proteins. ${ }^{\# \#} \mathrm{P}<0.01$ vs. control group; ${ }^{* *} \mathrm{P}<0.01$ vs. osteoporosis model group. Control, control group; Ost model, secondary osteoporosis model group; EGCG, epigallocatechin-3-gallate group; Runx2, runt-related transcription factor 2; OSX, Sp7 transcription factor osterix; PPAR $\gamma$, peroxisome proliferator-activated receptor $\gamma$.

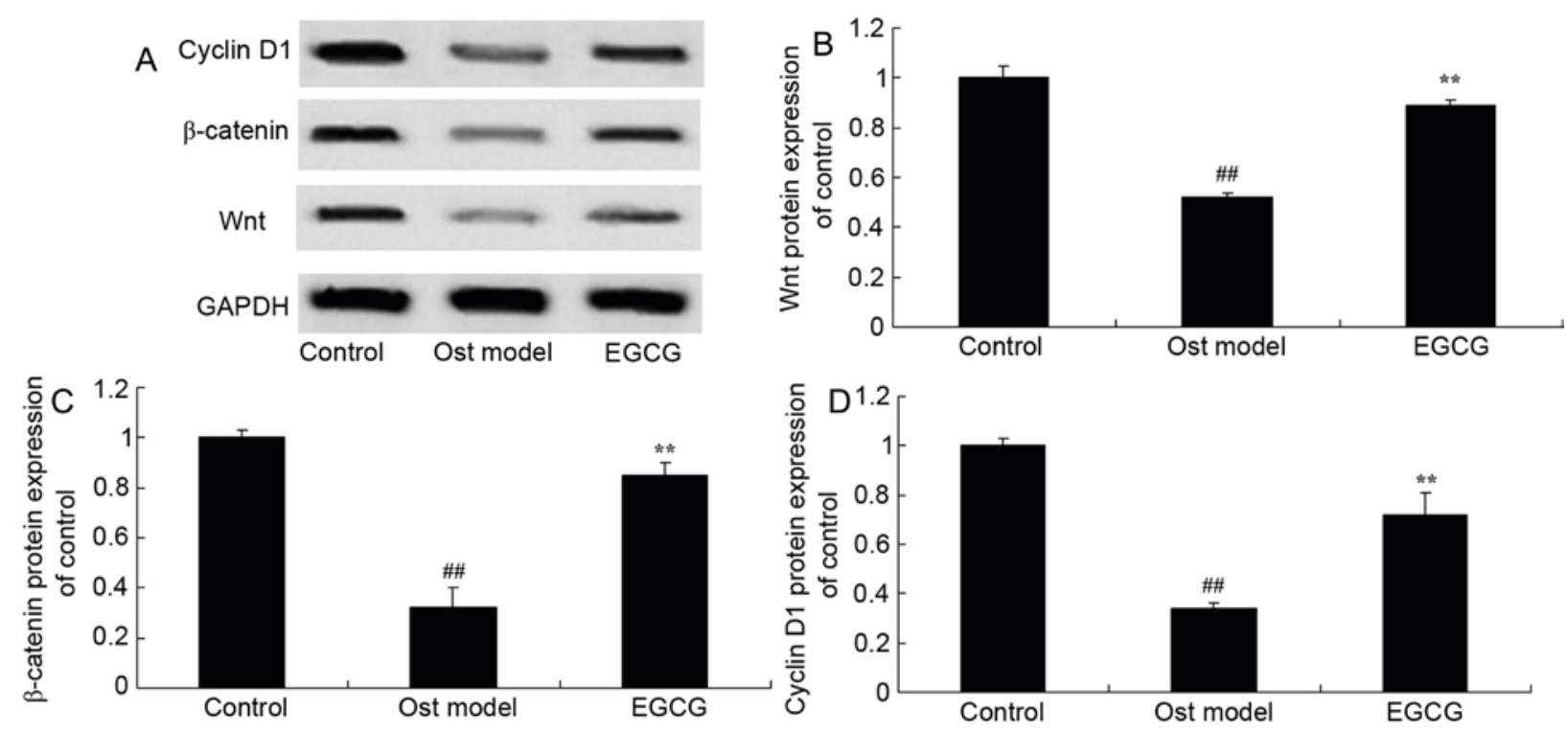

Figure 7. EGCG and Wnt, $\beta$-catenin and Cyclin D1 protein expression. EGCG protects against Wnt protein expression as shown by (A) western blot analysis. (B) Wnt, (C) $\beta$-catenin and (D) Cyclin D1 protein expression decreased in the mouse model of secondary osteoporosis, however, treatment with EGCG reversed this effect and increased the expression of these proteins. ${ }^{\sharp \#} \mathrm{P}<0.01$ vs. control group; ${ }^{* *} \mathrm{P}<0.01$ vs. osteoporosis model group. Control, control group; Ost model, secondary osteoporosis model group; EGCG, epigallocatechin-3-gallate group.

D1 protein expression in mice with secondary osteoporosis. Wang et al (33) reported that EGCG treatment protects against the hydrogen peroxide-induced inhibition of osteogenic differentiation through $\beta$-catenin and cyclin D1 expression in human bone marrow-derived mesenchymal stem cells.

In conclusion, the results of the present study indicated that EGCG treatment may be protective against lipid metabolism, secondary osteoporosis, and bone structure and turnover in mice with secondary osteoporosis induced by dexamethasone, as indicated by the analysis of PPAR $\gamma$ and the Wnt/ $\beta$-catenin signaling pathway. These results suggest that PPAR $\gamma$ and $\mathrm{Wnt} / \beta$-catenin may be novel therapeutic targets for future treatments of secondary osteoporosis using EGCG.

\section{Acknowledgements}

Not applicable. 


\section{Funding}

No funding was received.

\section{Availability of data and materials}

The analyzed data sets generated during the study are available from the corresponding author on reasonable request.

\section{Authors' contributions}

QL designed the experiment; JX, XL, JL, LG, HX and GW performed the experiments; QL and JX analyzed the data; QL wrote the manuscript.

\section{Ethics approval and consent to participate}

The animal protocol was approved by the Committee on the Ethics of Animal Experiments of the 309th Hospital of The People's Liberation Army (Beijing, China).

\section{Patient consent for publication}

Not applicable.

\section{Competing interests}

The authors declare that they have no competing interests.

\section{References}

1. Cohen A, Kamanda-Kosseh M, Recker RR, Lappe JM, Dempster DW, Zhou H, Cremers S, Bucovsky M, Stubby J and Shane E: Bone density after teriparatide discontinuation in premenopausal idiopathic osteoporosis. J Clin Endocrinol Metab 100: 4208-4214, 2015.

2. Nishiyama KK, Cohen A, Young P, Wang J, Lappe JM, Guo XE, Dempster DW, Recker RR and Shane E: Teriparatide increases strength of the peripheral skeleton in premenopausal women with idiopathic osteoporosis: A pilot HR-pQCT study. J Clin Endocrinol Metab 99: 2418-2425, 2014.

3. Frediani B, Bertoldi I, Pierguidi S, Nicosia A, Picerno V, Filippou G, Cantarini L and Galeazzi M: Improved efficacy of intramuscular weekly administration of clodronate $200 \mathrm{mg}$ (100 mg twice weekly) compared with $100 \mathrm{mg}$ (once weekly) for increasing bone mineral density in postmenopausal osteoporosis Clin Drug Investig 33: 193-198, 2013.

4. Leslie WD, Miller N, Rogala L and Bernstein CN: Body mass and composition affect bone density in recently diagnosed inflammatory bowel disease: The Manitoba IBD Cohort Study. Inflamm Bowel Dis 15: 39-46, 2009.

5. Jiang Y, Gou H, Wang S, Zhu J, Tian S and Yu L: Effect of pulsed electromagnetic field on bone formation and lipid metabolism of glucocorticoid-induced osteoporosis rats through canonical Wnt signaling pathway. Evid Based Complement Alternat Med 2016: 4927035, 2016.

6. Hampson G, Edwards S, Conroy S, Blake GM, Fogelman I and Frost ML: The relationship between inhibitors of the Wnt signalling pathway (Dickkopf-1(DKK1) and sclerostin), bone mineral density, vascular calcification and arterial stiffness in post-menopausal women. Bone 56: 42-47, 2013.

7. Colaianni G, Brunetti G, Faienza MF, Colucci S and Grano M: Osteoporosis and obesity: Role of Wnt pathway in human and murine models. World J Orthop 5: 242-246, 2014.

8. Meng J, Ma X, Wang N, Jia M, Bi L, Wang Y, Li M, Zhang H, Xue X, Hou Z, et al: Activation of GLP-1 receptor promotes bone marrow stromal cell osteogenic differentiation through $\beta$-catenin. Stem Cell Reports 6: 579-591, 2016.
9. Zhang JF, Li G, Chan CY, Meng CL, Lin MC, Chen YC, He ML, Leung PC and Kung HF: Flavonoids of Herba Epimedii regulate osteogenesis of human mesenchymal stem cells through BMP and Wnt/beta-catenin signaling pathway. Mol Cell Endocrinol 314: 70-74, 2010.

10. Liou SF, Hsu JH, Chu HC, Lin HH, Chen IJ and Yeh JL: KMUP-1 promotes osteoblast differentiation through cAMP and cGMP pathways and signaling of BMP-2/Smad1/5/8 and Wnt/ $\beta$-catenin. J Cell Physiol 230: 2038-2048, 2015.

11. Renaud J, Nabavi SF, Daglia M, Nabavi SM and Martinoli MG: Epigallocatechin-3-gallate, a promising molecule for parkinson's disease? Rejuvenation Res 18: 257-269, 2015.

12. Pae M and Wu D: Immunomodulating effects of epigallocatechin-3-gallate from green tea: Mechanisms and applications. Food Funct 4: 1287-1303, 2013

13. Peter B, Bosze S and Horvath R: Biophysical characteristics of proteins and living cells exposed to the green tea polyphenol epigallocatechin-3-gallate (EGCg): Review of recent advances from molecular mechanisms to nanomedicine and clinical trials. Eur Biophys J 46: 1-24, 2017.

14. Oliveira MR, Nabavi SF, Daglia M, Rastrelli L and Nabavi SM: Epigallocatechin gallate and mitochondria-A story of life and death. Pharmacol Res 104: 70-85, 2016.

15. Li X, Liu Y, Shi W, Xu H, Hu H, Dong Z, Zhu G, Sun Y, Liu B, Gao H, et al: Droplet digital PCR improved the EGFR mutation diagnosis with pleural fluid samples in non-small-cell lung cancer patients. Clin Chim Acta 471: 177-184, 2017.

16. Feagan BG, Sandborn WJ, Gasink C, Jacobstein D, Lang Y, Friedman JR, Blank MA, Johanns J, Gao LL, Miao Y, et al: Ustekinumab as induction and maintenance therapy for Crohn's disease. N Engl J Med 375: 1946-1960, 2016.

17. Livak KJ and Schmittgen TD: Analysis of relative gene expression data using real-time quantitative PCR and the 2(-Delta Delta C(T)) method. Methods 25: 402-408, 2001

18. Halse J, Greenspan S, Cosman F, Ellis G, Santora A, Leung A, Heyden N, Samanta S, Doleckyj S, Rosenberg E and Denker AE: A phase 2, randomized, placebo-controlled, dose-ranging study of the calcium-sensing receptor antagonist MK-5442 in the treatment of postmenopausal women with osteoporosis. J Clin Endocrinol Metab 99: E2207-E2215, 2014.

19. Liu HF, He HC, Yang L, Yang ZY, Yao K, Wu YC, Yang XB and He CQ: Pulsed electromagnetic fields for postmenopausal osteoporosis and concomitant lumbar osteoarthritis in southwest China using proximal femur bone mineral density as the primary endpoint: Study protocol for a randomized controlled trial. Trials 16: 265, 2015

20. Yan Z, Guo Y, Wang Y, Li Y and Wang J: MicroRNA profiles of BMSCs induced into osteoblasts with osteoinductive medium. Exp Ther Med 15: 2589-2596, 2018.

21. Marupanthorn K, Tantrawatpan C, Kheolamai P, Tantikanlayaporn D and Manochantr S: Bone morphogenetic protein-2 enhances the osteogenic differentiation capacity of mesenchymal stromal cells derived from human bone marrow and umbilical cord. Int J Mol Med 39: 654-662, 2017.

22. Ge W, Shi L, Zhou Y, Liu Y, Ma GE, Jiang Y, Xu Y, Zhang X and Feng $\mathrm{H}$ : Inhibition of osteogenic differentiation of human adipose-derived stromal cells by retinoblastoma binding protein 2 repression of RUNX2-activated transcription. Stem Cells 29: 1112-1125, 2011.

23. Ge C, Cawthorn WP, Li Y, Zhao G, Macdougald OA and Franceschi RT: Reciprocal control of osteogenic and adipogenic differentiation by ERK/MAP kinase phosphorylation of Runx2 and PPAR $\gamma$ transcription factors. J Cell Physiol 231: 587-596, 2016.

24. Hasegawa T, Oizumi K, Yoshiko Y, Tanne K, Maeda N and Aubin JE: The PPAR $\gamma$-selective ligand BRL-49653 differentially regulates the fate choices of rat calvaria versus rat bone marrow stromal cell populations. BMC Dev Biol 8: 71, 2008.

25. Duque G, Li W, Vidal C, Bermeo S, Rivas D and Henderson J: Pharmacological inhibition of PPAR $\gamma$ increases osteoblastogenesis and bone mass in male C57BL/6 mice. J Bone Miner Res 28: 639-648, 2013.

26. Lee $\mathrm{H}, \mathrm{Bae} \mathrm{S}$ and Yoon $\mathrm{Y}$ : The anti-adipogenic effects of (-) epigallocatechin gallate are dependent on the WNT/ $\beta$-catenin pathway. J Nutr Biochem 24: 1232-1240, 2013.

27. Xu S, Zhang Y, Liu B, Li K, Huang B, Yan B, Zhang Z, Liang K, Jia C, Lin J, et al: Activation of mTORC1 in B lymphocytes promotes osteoclast formation via regulation of $\beta$-catenin and RANKL/OPG. J Bone Miner Res 31: 1320-1333, 2016. 
28. Wang F, Wang Y, Zhao Y, Zhan Q, Yu P, Wang J and Xue C: Sialoglycoprotein isolated from eggs of carassius auratus ameliorates osteoporosis: An effect associated with regulation of the Wnt $/ \beta$-catenin pathway in rodents. J Agric Food Chem 64: $2875-2882,2016$

29. Zheng $\mathrm{X}, \mathrm{Wu} \mathrm{G}, \mathrm{Nie} \mathrm{Y}$ and Lin Y: Electroacupuncture at the governor vessel and bladder meridian acupoints improves postmenopausal osteoporosis through osteoprotegerin/RANKL/RANK and Wnt/ק-catenin signaling pathways. Exp Ther Med 10: 541-548, 2015.

30. Yun HM, Park KR, Quang TH, Oh H, Hong JT, Kim YC and Kim EC: 2,4,5-Trimethoxyldalbergiquinol promotes osteoblastic differentiation and mineralization via the BMP and Wnt/ß-catenin pathway. Cell Death Dis 6: e1819, 2015.
31. Fujita M, Urano T, Horie K, Ikeda K, Tsukui T, Fukuoka H, Tsutsumi O, Ouchi $\mathrm{Y}$ and Inoue S: Estrogen activates cyclin-dependent kinases 4 and 6 through induction of cyclin D in rat primary osteoblasts. Biochem Biophys Res Commun 299: 222-228, 2002

32. Hosking SM, Dobbins AG, Pasco JA and Brennan SL: Knowledge change regarding osteoporosis prevention: Translating recommended guidelines into user-friendly messages within a community forum. BMC Res Notes 8: 33, 2015.

33. Wang D, Wang Y, Xu S, Wang F, Wang B, Han K, Sun D and Li L: Epigallocatechin-3-gallate protects against hydrogen peroxide-induced inhibition of osteogenic differentiation of human bone marrow-derived mesenchymal stem cells. Stem Cells Int 2016: 7532798, 2016. 